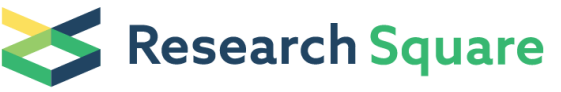 \\ Preprints are preliminary reports that have not undergone peer review. \\ They should not be considered conclusive, used to inform clinical practice, or referenced by the media as validated information.
}

\section{Alterations in Gamma-Delta T Cells in Patients With Primary Biliary Cholangitis}

\section{Sha Chen}

Capital Medical University Affiliated Beijing Friendship Hospital

Tingting Lv

Capital Medical University Affiliated Beijing Friendship Hospital

\section{Guangyong Sun}

Capital Medical University Affiliated Beijing Friendship Hospital

\section{Shuxiang Li}

Capital Medical University Affiliated Beijing Friendship Hospital

\section{Weijia Duan}

Capital Medical University Affiliated Beijing Friendship Hospital

\section{Chunpan Zhang}

Capital Medical University Affiliated Beijing Friendship Hospital Hua Jin

Capital Medical University Affiliated Beijing Friendship Hospital

\section{Dan Tian}

Capital Medical University Affiliated Beijing Friendship Hospital

\section{Mingyang Li}

Capital Medical University Affiliated Beijing Friendship Hospital

\section{Shan Shan}

Capital Medical University Affiliated Beijing Friendship Hospital

\section{Hong Ma}

Capital Medical University Affiliated Beijing Friendship Hospital

\section{Xiaojuan Ou}

Capital Medical University Affiliated Beijing Friendship Hospital

\section{Hong You}

Capital Medical University Affiliated Beijing Friendship Hospital

\section{Dong Zhang}

Capital Medical University Affiliated Beijing Friendship Hospital

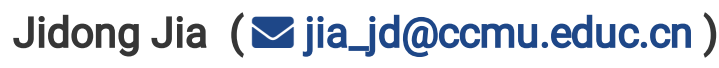

Beijing Friendship Hospital, Capital Medical Univerisity https://orcid.org/0000-0002-4673-8890

\section{Research Article}


Keywords: $\gamma \delta$ T cells, unconventional T cells, Vס2, TCR, primary biliary cirrhosis, autoimmune liver disease, subset, subpopulation, function, CXCR6, granzyme B

Posted Date: September 21st, 2021

DOI: https://doi.org/10.21203/rs.3.rs-860724/v1

License: (c) (1) This work is licensed under a Creative Commons Attribution 4.0 International License. Read Full License 


\section{Abstract \\ Background \& Aims}

Gamma-delta $(\mathrm{\gamma} \delta)$ T cells are involved in the development of diverse liver and autoimmune diseases, whereas the role of $y \delta T$ cells in primary biliary cholangitis (PBC) remains unclear.

\section{Methods}

We analyzed the number, phenotypes, and functional molecules of $\gamma \delta$ T cells in PBC patients $(n=74)$ and sex- and age-matched healthy controls (HCs) $(n=74)$ by flow cytometric analysis.

\section{Results}

We identified two distinct functional subsets of circulating $\gamma \delta T$ cells according to the CD3/TCRy $\delta$ complex: the TCRy $\delta^{\text {high }}$ and TCRy $\delta^{\text {low }}$ subsets. Approximately three-quarters of cells in the TCRy $\delta^{\text {high }}$

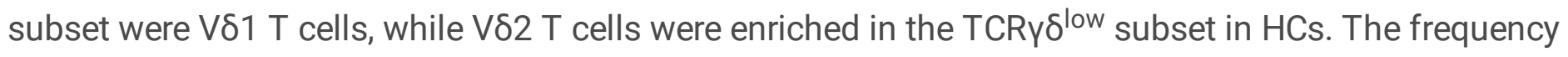
and absolute number of circulating TCRy ${ }^{\text {low }}$ cells was significantly decreased in PBC patients compared with HCs $(p<0.001)$. Furthermore, the frequency of TCRy $\delta^{\text {low }}$ cells was negatively correlated with disease severity and positively correlated with the ursodeoxycholic acid response. TCRy $\delta^{\text {low }}$ cells exhibited a similar apoptotic and proliferative phenotype but enhanced liver-homing chemokine receptor (CXCR6) expression in PBC patients compared with HCs. In addition, both TCRy $\delta^{\text {high }}$ and TCRy $\delta^{\text {low }}$ subsets were more activated in PBC compared with HCs, characterized by elevated expression levels of CD69 and HLADR. Finally, we found an increased granzyme B (GZMB) production and similar IFN- $y$ and TNF-a production of TCRy $\delta^{\text {low }}$ cells in PBC patients compared with HCs.

\section{Conclusion}

The TCRy $\delta^{\text {low }}$ subset might be a potential marker for disease progression and treatment response in PBC, which may play a crucial role in liver injury through increased CXCR6 expression and GZMB production.

\section{Introduction}

Primary biliary cholangitis (PBC) is an autoimmune liver disease with an increasing tendency of prevalence worldwide[1]. Although the pathogenesis of PBC has not been fully clarified, innate and adaptive immune responses play a key role in liver injury[2].

Gamma-delta $(\gamma \delta)$ T cells are a unique population of unconventional T cells with $\mathrm{y}$ and $\delta$ glycoprotein chains. In humans, $\gamma \delta T$ cells represent $2-10 \%$ of $T$ cells in the peripheral blood and $5-15 \%$ of $T$ cells in 
the liver, which regulates inflammation, pathogen clearance, and tumor immunity through the production of various cytokines[3-7]. Previous studies have demonstrated that $\gamma \delta T$ cells are closely involved in the development of viral hepatitis and autoimmune liver diseases $[5,8,9]$. However, the frequency of $\gamma \delta T$ cells in PBC remains controversial in previous studies with small sample sizes[9-12]. The function of $\gamma \delta$ $T$ cells in PBC also needs to be further explored.

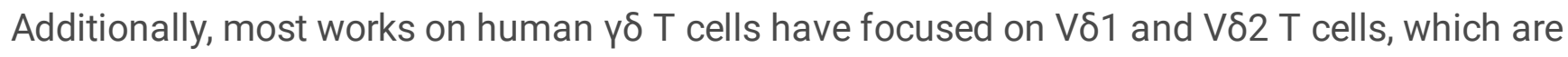
discriminated by different types of $\mathrm{Y}$ and $\delta$ chains[4]. Yokobori $\mathrm{N}$ et al. reported a novel way to identify two distinct subsets of $\gamma \delta T$ cells, called the TCRy $\delta^{\text {high }}$ and TCRy $\delta^{\text {low }}$ subsets, in patients with tuberculous pleurisy according to CD3/TCRy $\delta$ complex expression[13]. In line with this, a recent study found different expression levels of transcription factors, including PLZF and RORyt, between human circulating TCRy $\delta^{\text {high }}$ and TCRy $\delta^{\text {low }}$ cells[14], indicating that the two distinct subsets might have different phenotypes and functions. However, the role of the TCRy $\delta^{\text {high }}$ and TCRy $\delta^{\text {low }}$ subsets in PBC remains unclear.

Therefore, our study aimed to explore the number, phenotype, and functional molecules of $\gamma \delta \mathrm{T}$ cells in PBC, with a special focus on the TCRy $\delta^{\text {high }}$ and TCRy $\delta^{\text {low }}$ subsets.

\section{Patients And Methods}

\section{Patients}

PBC patients were diagnosed and enrolled at Beijing Friendship Hospital, Capital Medical University. This study was approved by the Institutional Committee for Human Research of Beijing Friendship Hospital, and written informed consent was obtained from all participants.

Patients will be included if they (1) were diagnosed with PBC based on international diagnostic criteria[2, 15 ] and (2) were treatment-naïve or received monotherapy with UDCA at a dose of $13-15 \mathrm{mg} / \mathrm{kg} / \mathrm{day}$. Patients were excluded if they (1) had coexistent other liver diseases, such as autoimmune hepatitis, chronic hepatitis B or C infection, drug-induced liver injury, alcoholic liver disease or metabolic fatty liver disease; (2) had coexistent malignant tumors, diabetes, or other autoimmune diseases, such as Sjogren's syndrome or autoimmune thyroiditis; or (3) were treated with corticosteroid or immunosuppressive drugs in the last 4 weeks.

Healthy controls (HCs) were included if they (1) were age- and sex-matched with PBC patients and (2) had normal routine blood, liver and kidney function, glucose, and lipid test results, routine urine test results, and abdominal ultrasound results in the last six months. HCs were excluded if they (1) had coexistent liver diseases, autoimmune diseases, diabetes, tumors or other diseases that can shorten life expectancy or (2) suffered from alcohol abuse.

\section{Blood sample collection and PBMC preparation}


Blood samples were obtained from 74 PBC patients and 74 healthy volunteers. Peripheral blood mononuclear cells (PBMCs) were freshly isolated by Ficoll-Hypaque density centrifugation.

\section{Analysis of the number and phenotypes of $\gamma \delta \mathrm{T}$ cells}

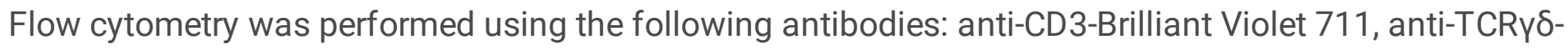
PE, anti-TCRV82-FITC, anti-CXCR6-PE/Cy7, anti-CXCR3-PE/Cy7, anti-CD69- PE/Cy7, anti-HLA-DRAPC/Cy7, anti-CD25- PE/Cy7, anti-Annexin V (AV)-FITC, and anti-Ki67-APC, which were purchased from Biolegend. Anti-TCRV81-PE/Cy7 was obtained from Thermo Scientific (Waltham, MA, USA).

Flow cytometry was performed using a FACSAria II flow cytometer (BD Biosciences, CA, USA), and the data were analyzed with FlowJo software (Treestar, Ashland, OR, USA).

\section{Detection of the functional molecules of $\gamma \delta \mathrm{T}$ cells}

For the detection of cytokines, PBMCs were cultured in complete medium (RPMI 1640, 10-040-CV, Corning, supplemented with $100 \mathrm{U} / \mathrm{mL}$ penicillin, $100 \mu \mathrm{g} / \mathrm{mL}$ streptomycin, $10 \% \mathrm{FBS}$, and $2 \mathrm{mM}$ glutamine) with phorbol myristate acetate (PMA)-ionomycin cocktail (Biolegend) at $50 \mathrm{ng} / \mathrm{ml}$ for 4 hours at $37^{\circ} \mathrm{C}$. Antibodies (anti-GZMB-FITC, anti-TNF-a-APC, and anti-IFN- $\gamma-P E / C y 7$ ) were purchased from Biolegend.

\section{Statistical analysis}

Categorical variables are expressed as counts and percentages. Continuous variables are all described as medians with interquartile ranges since they did not fit the normal distribution. The Mann-Whitney U-test was performed to compare the differences in continuous variables between PBC patients and HCs. The Wilcoxon matched-pairs signed rank test was performed to compare the differences between TCRy $\delta^{\text {high }}$ and TCRy $\delta^{\text {low }}$ cells. The Kruskal-Wallis tests was performed to compare the differences among treatment-naïve patients, nonresponders and responders. The chi-squared test was applied to compare the categorical variables between PBC patients and HCs. A two-sided P value $<0.05$ was considered significant. The analyses were conducted by using SPSS statistics version 26 .

\section{Results}

\section{Demographic and clinical characteristics of the subjects}

The demographic and clinical characteristics of PBC patients and HCs are shown in Table 1. A total of 74 PBC patients and 74 age- and sex-matched HCs were enrolled in the study. Significant differences were observed in serum levels of alkaline phosphatase (ALP), gamma-glutamyl transpeptidase (GGT), transaminase, albumin (ALB), total bilirubin (TBIL) and platelet count (PLT) between PBC patients and HCs. Among PBC patients, 12 were treatment-naïve, and 33 (44.6\%) responded to UDCA therapy according to Paris I (for cirrhotic PBC patients) and Paris II criteria (for noncirrhotic PBC patients). 
Table 1

Demographic and clinical characteristics of the subjects.

\begin{tabular}{|c|c|c|c|}
\hline Characteristics & $\mathrm{PBC}(\mathrm{n}=74)$ & HCs $(n=74)$ & $P$ value \\
\hline Age, years & $57(48-66)$ & $54(48-62)$ & 0.052 \\
\hline Female gender, n (\%) & $67(90.5)$ & $68(91.2)$ & 1.000 \\
\hline ALP, U/L & $144(99-236)$ & $70(55-83)$ & $<0.001$ \\
\hline GGT, U/L & $67(36-140)$ & $14(13-18)$ & $<0.001$ \\
\hline$A L T, U / L$ & $29(18-44)$ & $15(13-20)$ & $<0.001$ \\
\hline AST, U/L & $33(27-50)$ & $20(17-22)$ & $<0.001$ \\
\hline ALB, g/L & $40.3(34.2-43.7)$ & 42.7 (40.9-44.8) & 0.001 \\
\hline TBIL, $\mu \mathrm{mol} / \mathrm{L}$ & $17.6(11.7-26.3)$ & $12.6(11.0-15.4)$ & $<0.001$ \\
\hline PLT, 10^9/L & $168(90-216)$ & $235(191-262)$ & $<0.001$ \\
\hline $\lg G, \mathrm{mg} / \mathrm{dl}$ & $1490(1270-1730)$ & l & / \\
\hline $\mathrm{lgM}, \mathrm{mg} / \mathrm{dl}$ & $227(122-355)$ & l & l \\
\hline AMA-M $_{2}(+), n(\%)$ & $64(86.5)$ & l & l \\
\hline Cirrhosis, n (\%) & $28(37.8)$ & / & / \\
\hline Treatment-naive, n (\%) & $12(16.2)$ & / & / \\
\hline UDCA responders", n (\%) & $33(44.6)$ & / & / \\
\hline \multicolumn{4}{|c|}{$P$ values refer to comparisons between PBC patients and healthy controls. } \\
\hline \multicolumn{4}{|c|}{$\begin{array}{l}\text { \#Paris I and Paris II criteria were performed in cirrhotic and non-cirrhotic PBC patients to identify UDC } \\
\text { responders, respectively. }\end{array}$} \\
\hline \multicolumn{4}{|c|}{$\begin{array}{l}\text { PBC, primary biliary cholangitis; HCs, healthy controls; ALP, alkaline phosphatase; GGT, gamma- } \\
\text { glutamyl transpeptidase; ALT, alanine aminotransferase; AST, aspartate aminotransferase; ALB, } \\
\text { albumin; TBIL, total bilirubin; PLT, platelet count; IgG, immunoglobulin G; IgM, immunoglobulin M; } \\
\text { AMA-M2, anti-mitochondrial M2 antibody; UDCA, ursodeoxycholic acid. }\end{array}$} \\
\hline
\end{tabular}

\section{Circulating TCRyd low cells were significantly decreased in PBC patients compared with HCs}

y $\delta$ T cells were defined as CD3 + TCRy $\delta$ + cells in the study. The representative flow cytometric analysis demonstrated two distinct subsets based on the CD3/yסTCR complex: the TCRy $\delta^{\text {high }}$ and TCRy $\delta^{\text {low }}$ subsets (Fig. 1a). The frequency of circulating TCRy $\delta^{\text {low }}$ cells among CD3 + cells was significantly higher than that of TCRy ${ }^{\text {high }}$ cells among CD3 + cells both in PBC patients (median $2.75 \%$ vs. $0.94 \%$ ) and HCs (median $5.84 \%$ vs. $0.88 \%$ ) (both $p<0.001$ ). 
The frequency and absolute number of circulating TCRy $\delta^{\text {low }}$ cells were significantly decreased in PBC patients $(n=74)$ compared with HCs $(n=74)(p<0.001)$, but the frequency and number of circulating TCRy $\delta^{\text {high }}$ cells were similar between the two groups $(p>0.05)$ (Fig. 1b).

\section{The frequency of circulating TCRy ${ }^{\text {low }}$ cells was correlated with disease severity and UDCA response}

We found a significant positive correlation between the frequency of circulating TCRy $\delta^{\text {low }}$ cells and the serum level of PLT $(p=0.001, r=0.396)$, but no correlations were found between the frequency of circulating TCRy $\delta^{\text {low }}$ cells and the levels of TBIL, ALP or aspartate aminotransferase (AST) (Fig. 1c). Furthermore, the frequency and absolute number of circulating TCRy $\delta^{\text {low }}$ cells were significantly decreased in cirrhotic PBC patients $(n=28)$ compared with noncirrhotic PBC patients $(n=46)($ Fig. 1 d).

Additionally, subgroup analysis showed that the frequency and absolute number of circulating TCRy $\delta^{\text {low }}$ cells were the lowest in UDCA treatment-naïve PBC patients, followed by nonresponders and responders. A significantly decreased frequency and number of circulating TCRY $\delta^{\text {low }}$ cells were detected in treatmentnaïve patients $(n=12)$ and UDCA nonresponders $(n=29)$ compared with UDCA responders $(n=33)$ (Fig. 1e, p < 0.05).

\section{The proportion of Vס2 T cells in the circulating TCRy ${ }^{\text {low }}$ subset was decreased in PBC patients}

We further detected the expression of $\mathrm{V} \delta 1$ and V $\delta 2$ in the TCRy $\delta^{\text {high }}$ and TCRy $\delta^{\text {low }}$ subsets (Fig. 2a). Approximately $75.1 \%$ of $\mathrm{TCR} \gamma \delta^{\text {high }}$ cells were $\mathrm{V} \delta 1 \mathrm{~T}$ cells and $19.3 \%$ were $\mathrm{V} \delta 1^{-} \mathrm{V} \delta 2^{-} \mathrm{T}$ cells in $\mathrm{HCs}$. The proportion of V $\delta 1 \mathrm{~T}$ cells in the TCRy $\delta^{\text {high }}$ subset was not different between HCs and PBC patients $(p>$ 0.05, Fig. 2b).

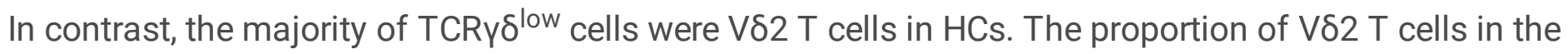
TCRy $\delta^{\text {low }}$ subset was decreased ( $89.4 \%$ vs. $\left.97.4 \%\right)$, and the proportion of $\mathrm{V} \delta 1^{-} \mathrm{V} \delta 2^{-} \mathrm{T}$ cells was increased (7.3\% vs. $2.1 \%)$ in PBC patients compared with HCs ( $p<0.001$, Fig. $2 \mathrm{c})$.

\section{Circulating TCRy $\delta^{\text {low }}$ cells exhibited similar apoptotic and proliferative phenotypes and enhanced CXCR6 expression in PBC patients}

To explain the reduction in TCRy $\delta^{\text {low }}$ cells in PBC patients, we assessed the apoptosis, proliferation, and expression of liver-homing chemokine receptors of $y \delta T$ cells (Fig. 3a). No difference was observed in the expression of annexin-V (AV) and Ki67 in either circulating TCRy $\delta^{\text {high }}$ cells or TCRy $\delta^{\text {low }}$ cells between HCs and PBC patients ( $p>0.05$, Fig. $3 b$ and $3 c$ ).

Compared with TCRy $\delta^{\text {high }}$ cells, the expression of liver-homing chemokine receptors, including CXCR6 and CXCR3, was significantly higher in TCRy $\delta^{\text {low }}$ cells in both HCs and PBC patients (all $p<0.001$ ). The expression of CXCR6 in TCRy $\delta^{\text {high }}$ cells and TCRy $\delta^{\text {low }}$ cells was significantly increased in PBC patients 
compared with HCs $(p<0.05$, Fig. $3 d)$, but the expression of CXCR3 in TCRy $\delta^{\text {high }}$ cells and TCRy $\delta^{\text {low }}$ cells was comparable between the two groups ( $p>0.05$, Fig. $3 e)$.

\section{Circulating TCRy $\delta^{\text {low }}$ cells demonstrated an activated phenotype and enhanced GZMB production in PBC patients}

The expression of activation markers, including CD69, HLA-DR, and CD25, in circulating y $\delta$ T cells was analyzed in PBC patients and HCs. Circulating TCRy ${ }^{\text {high }}$ cells were more activated than TCRy $\delta^{\text {low }}$ cells in both HCs and PBC patients. Both the circulating TCRy $\delta^{\text {high }}$ cells and TCRy $\delta^{\text {low }}$ cells in PBC were more activated than those in HCs, characterized by higher expression of CD69 and HLA-DR $(p<0.05$, Fig. 4a).

In addition, circulating TCRy $\delta^{\text {high }}$ cells produced higher GZMB but lower IFN- $\gamma$ and TNF-a than TCRy $\delta^{\text {low }}$ cells in both HCs and PBC patients. We found an increased GZMB production of TCRy ${ }^{\text {low }}$ cells in PBC patients $(\mathrm{n}=28)$ compared with HCs $(\mathrm{n}=30)(p<0.05$, Fig. $4 \mathrm{c})$. The production of IFN- $\gamma$ and TNF-a by TCRy $\delta^{\text {low }}$ cells in PBC patients was lower than that in $\mathrm{HCs}$, but the results remained statistically insignificant ( $p>0.05$, Fig. $4 c)$.

\section{Discussion}

In the present study, we analyzed the number, phenotype, and functional molecules of $\gamma \delta T$ cells in PBC patients and sex-and age-matched HCs by flow cytometric analysis. We found that the number and function of TCRy ${ }^{\text {low }}$ cells were significantly altered in $\mathrm{PBC}$ patients compared with $\mathrm{HCs}$, which might be involved in the pathogenesis of PBC.

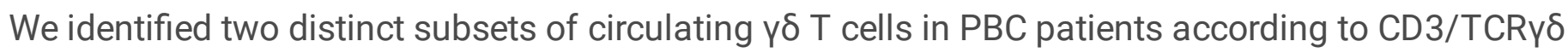
complex expression: the TCRy $\delta^{\text {high }}$ and TCRy $\delta^{\text {low }}$ subsets. Approximately three-quarters of cells in the

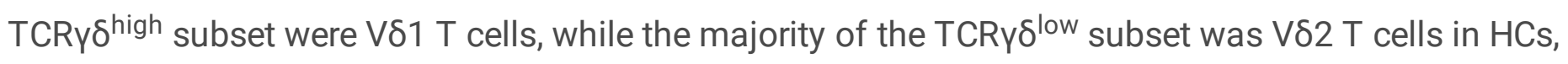
which was consistent with the results of a previous study[13]. Furthermore, these two subpopulations differed from each other in terms of the expression levels of chemokines, activation markers, and functional molecules. Compared with the TCRy $\delta^{\text {high }}$ subset, the TCRy $\delta^{\text {low }}$ subset expressed higher levels of liver-homing chemokine receptors, lower activation markers, lower GZMB, and higher IFN- $y$ and TNF-a.

The change in the quantity of circulating $y \delta T$ cells in PBC patients remains controversial in previous studies [9-12]. Studies have found a significantly decreased frequency of circulating $\gamma \delta T$ cells in PBC patients compared with $\mathrm{HCs}[11,12]$, whereas the other two studies showed a similar frequency of $\gamma \delta \mathrm{T}$ cells between the two groups $[9,10]$. This might be due to the small sample sizes and different disease stages. The number of patients in our study was more than twice that of the previous studies. We found that the frequency and the absolute number of $\gamma \delta T$ cells was significantly decreased in PBC patients compared with $\mathrm{HCs}$, and the $\gamma \delta \mathrm{T}$ cells that were decreased in number were the those in the circulating TCRy $\delta^{\text {low }}$ subset. The relatively large sample size allowed us to further analyze the frequency of TCRy $\delta^{\text {low }}$ cells in subgroups. The results showed that the frequency of TCRy $\delta^{\text {low }}$ cells was negatively 
correlated with disease severity and positively correlated with the UDCA response, indicating that TCRy $\delta^{\text {low }}$ cells might be a potential marker of disease progression and treatment response.

To explore the possible reasons for the reduction in TCRy $\delta^{\text {low }}$ cells in PBC patients, we further detected the apoptotic and proliferative phenotype and the expression of liver-homing chemokine receptors on $\gamma \delta$ $T$ cells. We found that circulating TCRy $\delta^{\text {low }}$ cells exhibited a similar apoptotic and proliferative phenotype but enhanced liver-homing chemokine receptor (CXCR6) expression in PBC patients compared with HCs. Therefore, we postulated that the reduction in TCRy $\delta^{\text {low }}$ cells might be caused by redistribution to the liver. In line with this hypothesis, studies found an increased number and frequency of hepatic $ү \delta \mathrm{T}$ cells in autoimmune liver diseases and chronic liver diseases compared with $\mathrm{HCs}[9,16,17]$. However, a recent study demonstrated a decreased frequency of hepatic $ү \delta \mathrm{T}$ cells in 13 PBC and other chronic liver diseases patients compared with that in patients with healthy livers. Of note, all 13 of these PBC patients had end-stage liver disease, which might be an explanation for the opposite results. Future studies with larger sample sizes and different disease stages are needed to explore the quantity and function of $y \delta T$ cells and their subpopulations in PBC livers.

Generally, the CXCR6-CXCL16 axis is considered to promote inflammation and disease progression in various liver diseases, including fibrosis[18], hepatocellular carcinoma[19], acute liver injury[20, 21], and nonalcoholic fatty liver disease[22]. Our results showed that approximately one-third of circulating TCRy ${ }^{\text {low }}$ cells expressed CXCR6, and the proportion was even higher in PBC patients than in HCs. Consistent with our results, a recent study using single-cell RNA sequencing found that CXCR6 is preferentially expressed by hepatic $ү \delta T$ cells[17]. Taken together, this evidence suggests that $\gamma \delta T$ cells might be involved in liver injury through the CXCR6-CXCL16 axis.

The function of $\gamma \delta$ T cells in PBC has not been well elucidated. Human $\gamma \delta$ T cells display antiviral, proinflammatory or cytotoxic abilities in different liver and autoimmune diseases through the production of various cytokines, mainly IFN- $\gamma$, TNF- $a$, and GZMB $[4,5,8,23]$. In this study, we analyzed the expression of activation markers and functional molecules of both the TCRy $\delta^{\text {high }}$ and TCRy $\delta^{\text {low }}$ subsets in PBC patients and HCs. We demonstrated that the circulating TCRy $\delta^{\text {low }}$ subset exhibited an activated phenotype and enhanced cytotoxicity characterized by increased GZMB production in PBC patients, suggesting that the TCRy $\delta^{\text {low }}$ subset might cause liver damage through GZMB production. Consistent with our results, Ferri $S$ et al. found that $\gamma \delta T$ cells produced higher GZMB in 47 patients with autoimmune hepatitis than in $28 \mathrm{HCs}$, and the production of GZMB was correlated with biochemistry variables of liver damage[8]. In addition, another study demonstrated that circulating Vס2 T cell function shifted from antiviral toward cytotoxicity after HCV infection, which is characterized by lower IFN-y but higher GZMB

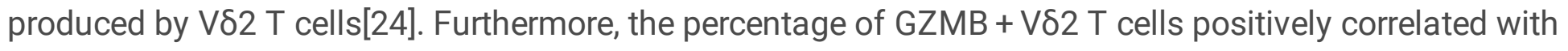
serum ALT levels in HCV-infected patients[24]. All these results showed that $ү \delta T$ cells might induce liver injury through enhanced GZMB production.

This study has several limitations. First, we failed to analyze the number and function of $\gamma \delta \mathrm{T}$ cells in the liver by immunohistology, and further relevant research on hepatic $\gamma \delta T$ cells by new strategies, such as 
RNA scope, is needed. Second, this is a cross-sectional study that enrolled patients with different disease stages and treatment responses. Long-term follow-up of the level of $ү \delta T$ cells in the same cohort before and after UDCA treatment will provide more value.

In summary, we identified two distinct functional subsets of circulating $y \delta T$ cells according to the

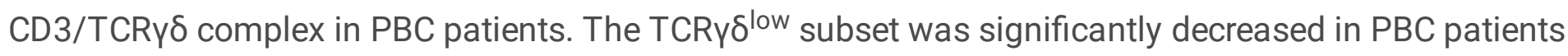
and correlated with disease severity and the UDCA response, and this subset may play a crucial role in liver injury through increased CXCR6 expression and GZMB production.

\section{Declarations}

Funding This work is funded by National Natural Science Foundation of China (82000533).

Conflicts of interest The authors declare that they have no conflict of interest.

Ethics approval All procedures followed were in accordance with the ethical standards of the responsible committee on human experimentation (institutional and national) and with the Helsinki Declaration of 1975, as revised in 2008. The ethics committee of the Beijing Friendship Hospital, Capital Medical University reviewed and approved the study.

Consent to participate Written informed consent was obtained from all subjects enrolled in the study.

Consent for publication Written informed consent for publication was obtained from all participants.

Availability of data and material Not applicable.

Code availability Not applicable.

Authors' contributions Professors Jidong Jia and Dong Zhang designed, revised and finalized the manuscript; Doctor Sha Chen performed the experiment, analyzed the data and drafted the manuscript; Doctors Tingting Lv, Chunpan Zhang, Hua Jin, Mingyang Li, Guangyong Sun and Dan Tian participated in performing and guiding the experiment; Doctors Shuxiang Li, Weijia Duan, Shan Shan, and Hong Ma collected the blood sample and clinical data; Professors Guangyong, Sun, Xiaojuan Ou, and Hong You critically reviewed and revised the manuscript. All authors read and approved the final manuscript.

\section{References}

1. Lv T, Chen S, Li M, et al. Regional variation and temporal trend of primary biliary cholangitis epidemiology: A systematic review and meta-analysis. J Gastroenterol Hepatol. 2020;36:1423-34.

2. European Association for the Study of the Liver. EASL Clinical Practice Guidelines: The diagnosis and management of patients with primary biliary cholangitis. J Hepatol. 2017;67:145-72.

3. Gao B, Jeong WI, Tian Z. Liver: An organ with predominant innate immunity. Hepatology. 2008;47:729-36. 
4. Lawand M, Dechanet-Merville J, Dieu-Nosjean MC. Key Features of Gamma-Delta T-Cell Subsets in Human Diseases and Their Immunotherapeutic Implications. Front Immunol. 2017;8:761.

5. Rajoriya N, Fergusson JR, Leithead JA, et al. Gamma Delta T-lymphocytes in Hepatitis C and Chronic Liver Disease. Frontiers in Immunology 2014;5.

6. Vantourout P, Hayday A. Six-of-the-best: unique contributions of gammadelta T cells to immunology. Nat Rev Immunol. 2013;13:88-100.

7. Kenna T, Golden-Mason L, Norris S, et al. Distinct subpopulations of gamma delta T cells are present in normal and tumor-bearing human liver. Clin Immunol. 2004;113:56-63.

8. Ferri S, Longhi MS, De Molo C, et al. A multifaceted imbalance of T cells with regulatory function characterizes type 1 autoimmune hepatitis. Hepatology. 2010;52:999-1007.

9. Martins EB, Graham AK, Chapman RW, et al. Elevation of gamma delta T lymphocytes in peripheral blood and livers of patients with primary sclerosing cholangitis and other autoimmune liver diseases. Hepatology. 1996;23:988-93.

10. Hua F, Wang L, Rong X, et al. Elevation of Vdelta1 T cells in peripheral blood and livers of patients with primary biliary cholangitis. Clin Exp Immunol. 2016;186:347-55.

11. Niehues T, Gulwani-Akolkar B, Goldman IS, et al. Marked gamma delta T-cell decrease in peripheral blood of patients with primary biliary cirrhosis (PBC). Autoimmunity. 1994;18:267-73.

12. Jang JS, Juran BD, Cunningham KY, et al. Single-cell mass cytometry on peripheral blood identifies immune cell subsets associated with primary biliary cholangitis. Sci Rep. 2020;10:12584.

13. Yokobori N, Schierloh P, Geffner L, et al. CD3 expression distinguishes two gammadeltaT cell receptor subsets with different phenotype and effector function in tuberculous pleurisy. Clin Exp Immunol. 2009;157:385-94.

14. Venken K, Jacques $\mathrm{P}$, Mortier $\mathrm{C}$, et al. RORgammat inhibition selectively targets IL-17 producing iNKT and gammadelta-T cells enriched in Spondyloarthritis patients. Nat Commun. 2019;10:9.

15. Lindor KD, Bowlus CL, Boyer J, et al. Primary Biliary Cholangitis: 2018 Practice Guidance from the American Association for the Study of Liver Diseases. Hepatology. 2019;69:394-419.

16. Kanayama K, Morise K, Nagura H. Immunohistochemical study of T cell receptor gamma delta cells in chronic liver disease. Am J Gastroenterol. 1992;87:1018-22.

17. Zhao J, Zhang S, Liu Y, et al. Single-cell RNA sequencing reveals the heterogeneity of liver-resident immune cells in human. Cell Discov. 2020;6:22.

18. Wehr A, Baeck C, Heymann F, et al. Chemokine receptor CXCR6-dependent hepatic NK T Cell accumulation promotes inflammation and liver fibrosis. J Immunol. 2013;190:5226-36.

19. Gao Q, Zhao YJ, Wang XY, et al. CXCR6 upregulation contributes to a proinflammatory tumor microenvironment that drives metastasis and poor patient outcomes in hepatocellular carcinoma. Cancer Res. 2012;72:3546-56.

20. Wehr A, Baeck C, Ulmer F, et al. Pharmacological inhibition of the chemokine CXCL16 diminishes liver macrophage infiltration and steatohepatitis in chronic hepatic injury. PLoS One. 2014;9:e112327. 
21. Wang $\mathrm{H}$, Shao $\mathrm{Y}$, Zhang S, et al. CXCL16 deficiency attenuates acetaminophen-induced hepatotoxicity through decreasing hepatic oxidative stress and inflammation in mice. Acta Biochim Biophys Sin (Shanghai). 2017;49:541-9.

22. Ma KL, Wu Y, Zhang Y, et al. Activation of the CXCL16/CXCR6 pathway promotes lipid deposition in fatty livers of apolipoprotein E knockout mice and HepG2 cells. Am J Transl Res. 2018;10:1802-16.

23. O'Brien RL, Born WK. Two functionally distinct subsets of IL-17 producing gammadelta T cells. Immunol Rev. 2020;298:10-24.

24. Yin W, Tong S, Zhang Q, et al. Functional dichotomy of Vdelta2 gammadelta T cells in chronic hepatitis $\mathrm{C}$ virus infections: role in cytotoxicity but not for IFN-gamma production. Sci Rep. 2016;6:26296.

\section{Figures}


a

Gated on CD3+

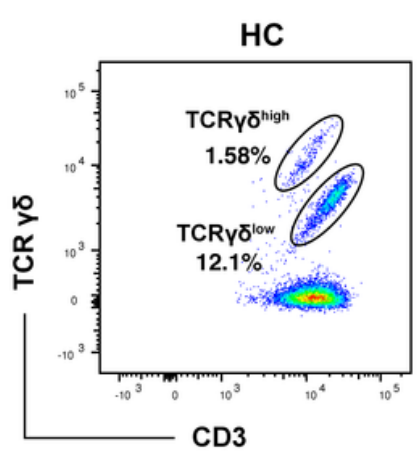

c
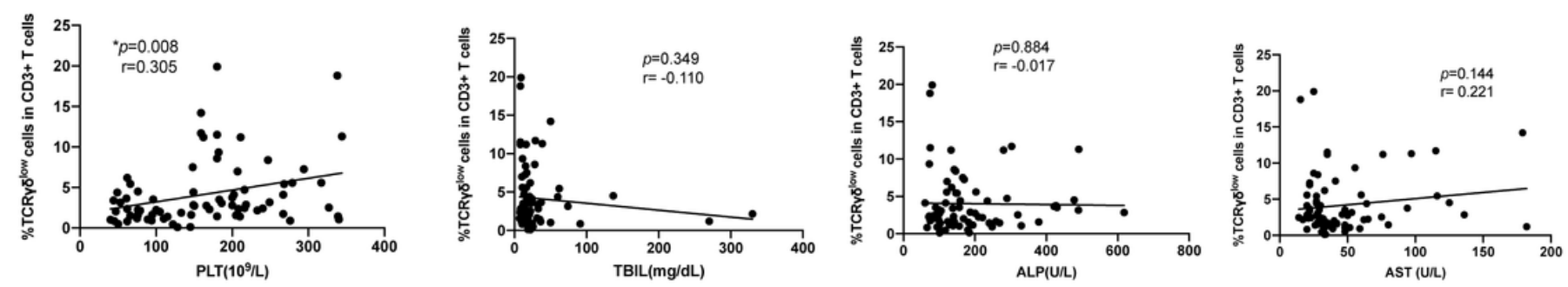

d

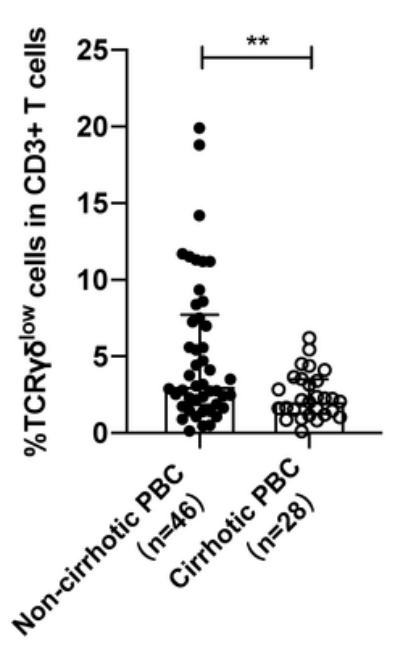

b
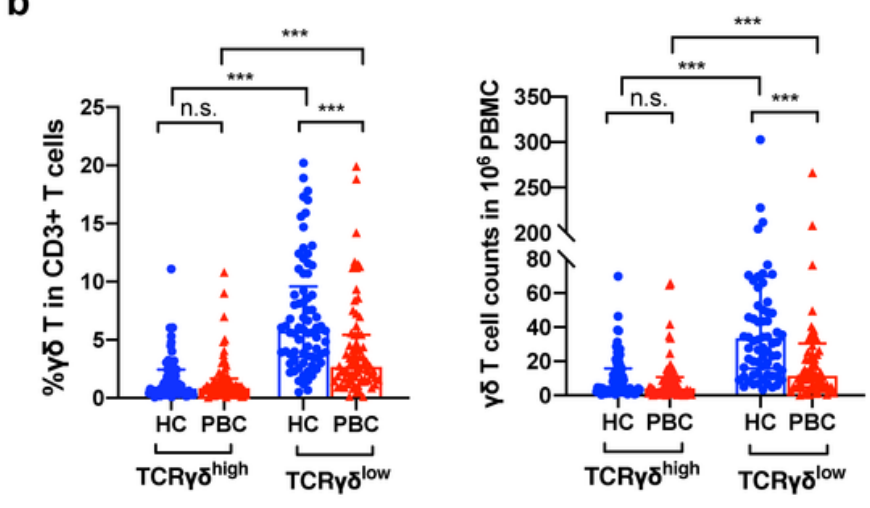
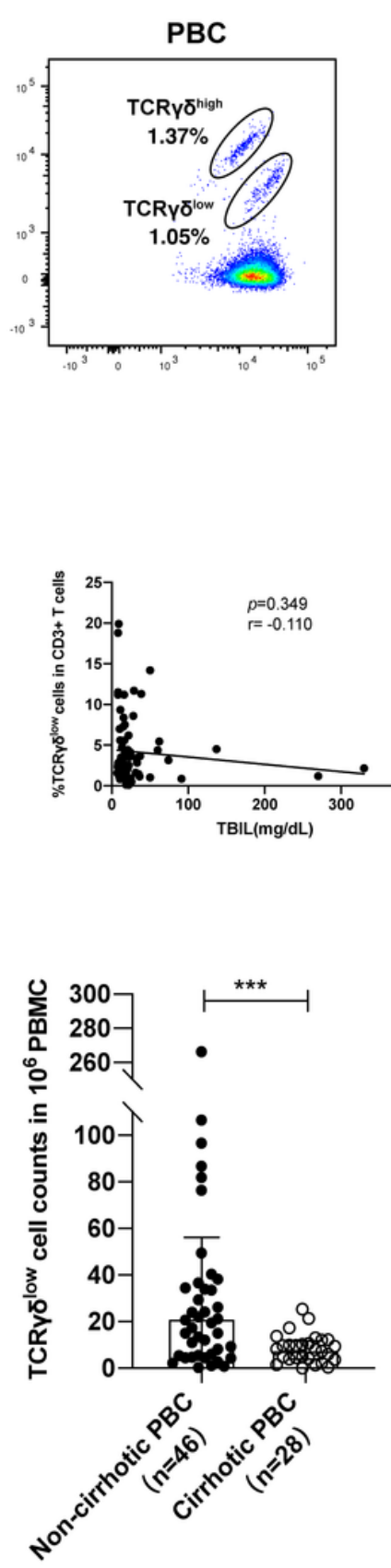

e
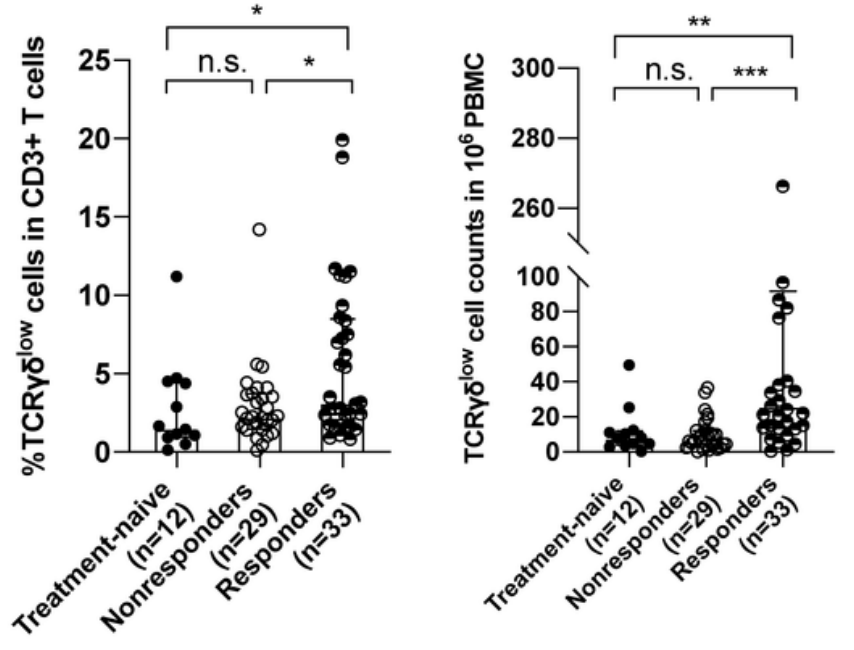

Figure 1

Decreased frequency and number of circulating TCRyסlow cells in PBC patients. (a) Representative flow cytometric analysis of circulating $\gamma \delta$ T cells in HCs and PBC patients. (b) Decreased frequencies (left) and numbers (right) of circulating TCRyठlow cells were detected in PBC patients $(n=74)$ compared with HCs $(n=74)$. (c) The frequency of circulating TCRyolow cells was positively correlated with PLT but not correlated with the levels of TBIL, ALP or AST ( $n=74)$. (d) Decreased frequencies (left) and numbers (right) of circulating TCRyolow cells were detected in cirrhotic PBC patients $(n=28)$ compared with noncirrhotic PBC patients $(n=46)$. (e) Decreased frequencies (left) and numbers (right) of circulating 
TCRyठlow cells were detected in treatment-naïve PBC patients $(n=12)$ and UDCA nonresponders $(n=29)$ compared with UDCA responders $(n=33)$. Data are presented as medians with interquartile ranges. ${ }^{*} \mathrm{P}$ $0.05,{ }^{*} \mathrm{P}<0.01, * \star \star \mathrm{P}<0.001$, n.s. $\mathrm{P}>0.05$. PBC, primary biliary cholangitis; $\mathrm{HCs}$, healthy controls; PLT, platelet count; TBIL, total bilirubin; ALP, alkaline phosphatase; AST, aspartate aminotransferase; UDCA, ursodeoxycholic acid.

a

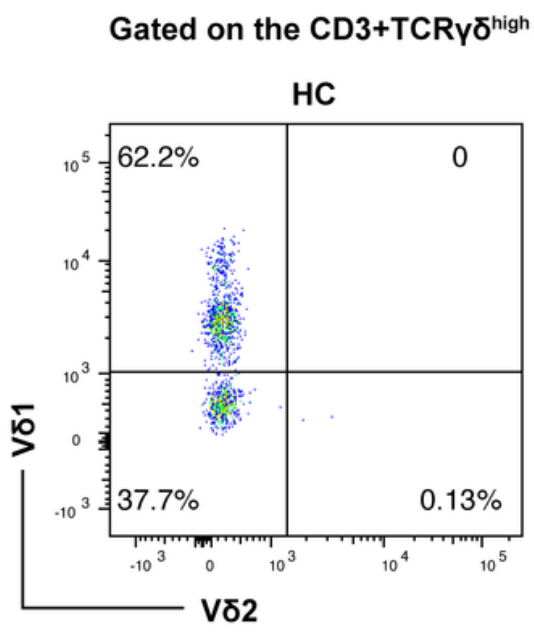

Gated on the CD3+TCRy $\delta^{\text {high }}$ subset

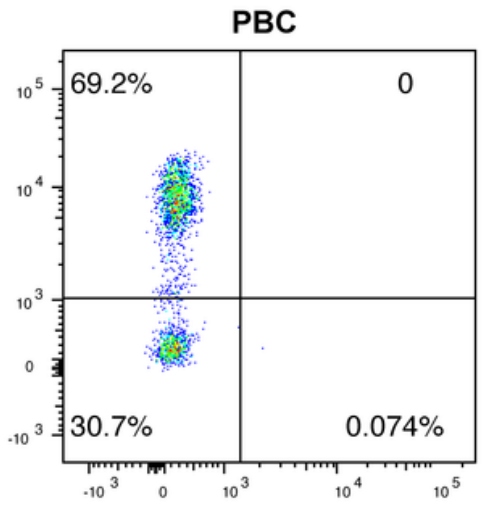

Gated on the CD3+TCRy $\delta^{\text {low }}$ subset

$\mathrm{HC}$
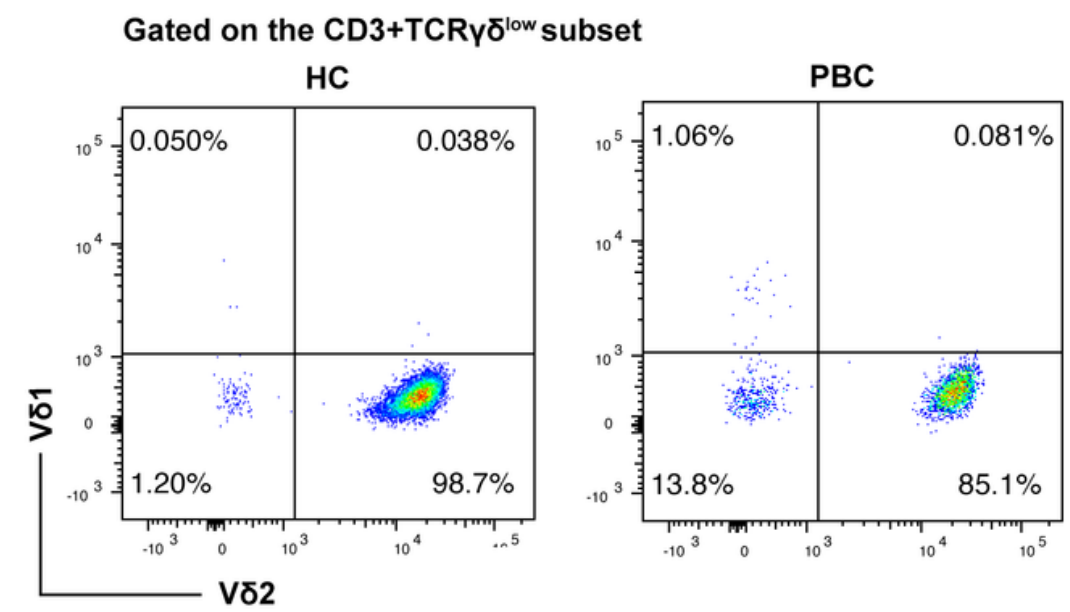

b

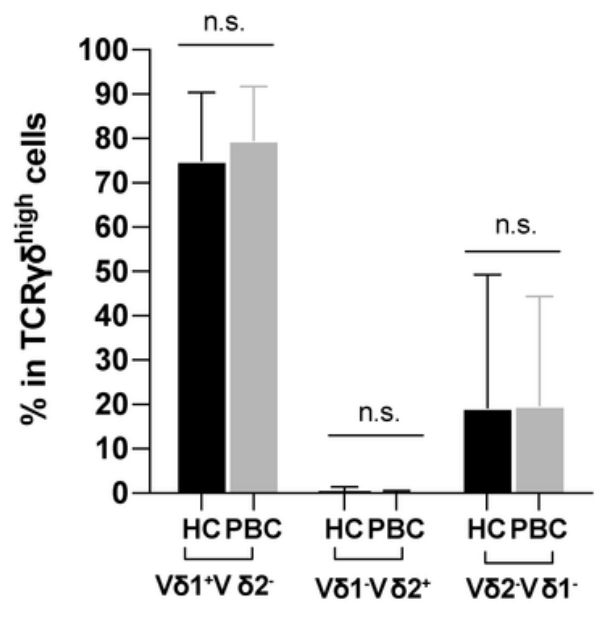

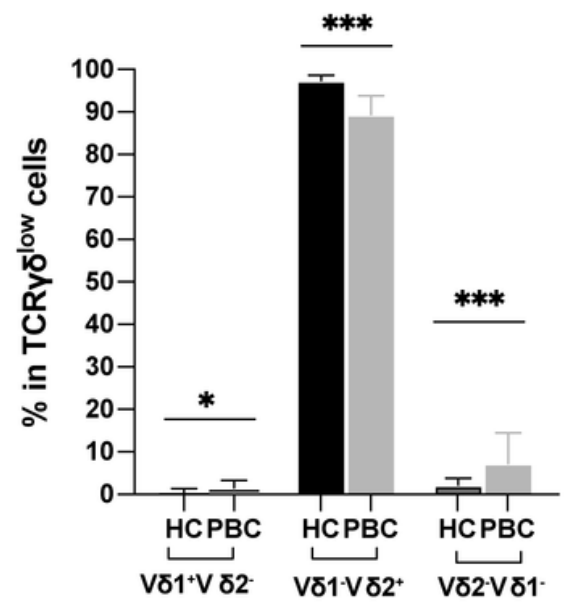

\section{Figure 2}

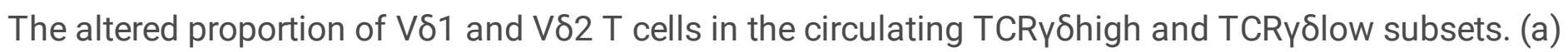

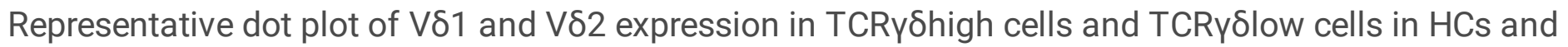

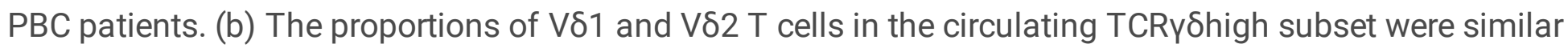
between HCs $(n=20)$ and PBC patients $(n=20)$. (c) A decreased proportion of Vס2 T cells and an increased

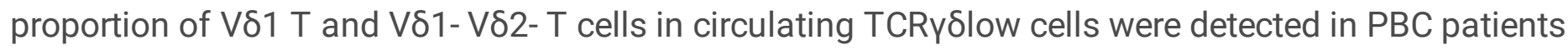
$(n=20)$ compared with HCs $(n=20)$. Data are presented as medians with interquartile ranges. ${ }^{*} P<0.05$, $\star \star P<0.01,{ }^{\star \star \star} P<0.001$, n.s. $P>0.05$. HCs, healthy controls; PBC, primary biliary cholangitis. 
a

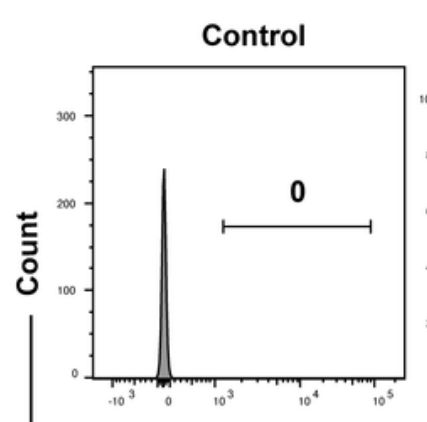

AV
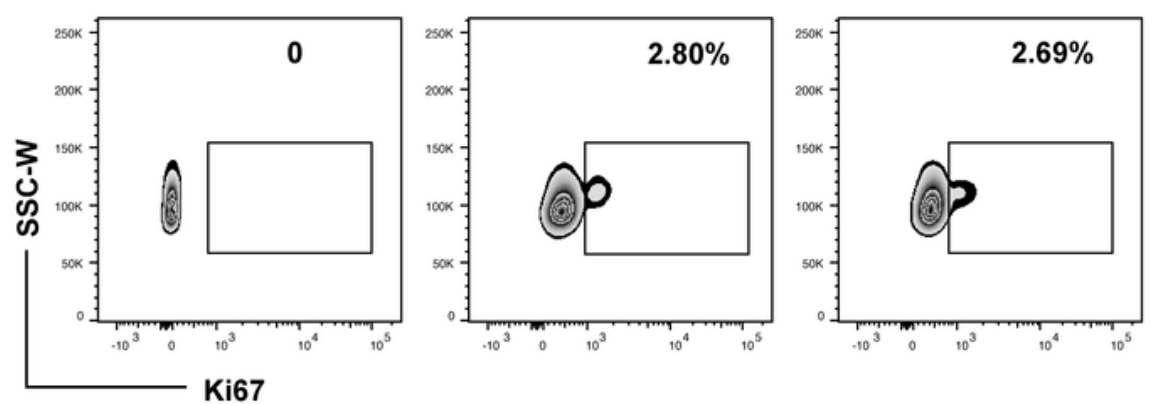

Ki67
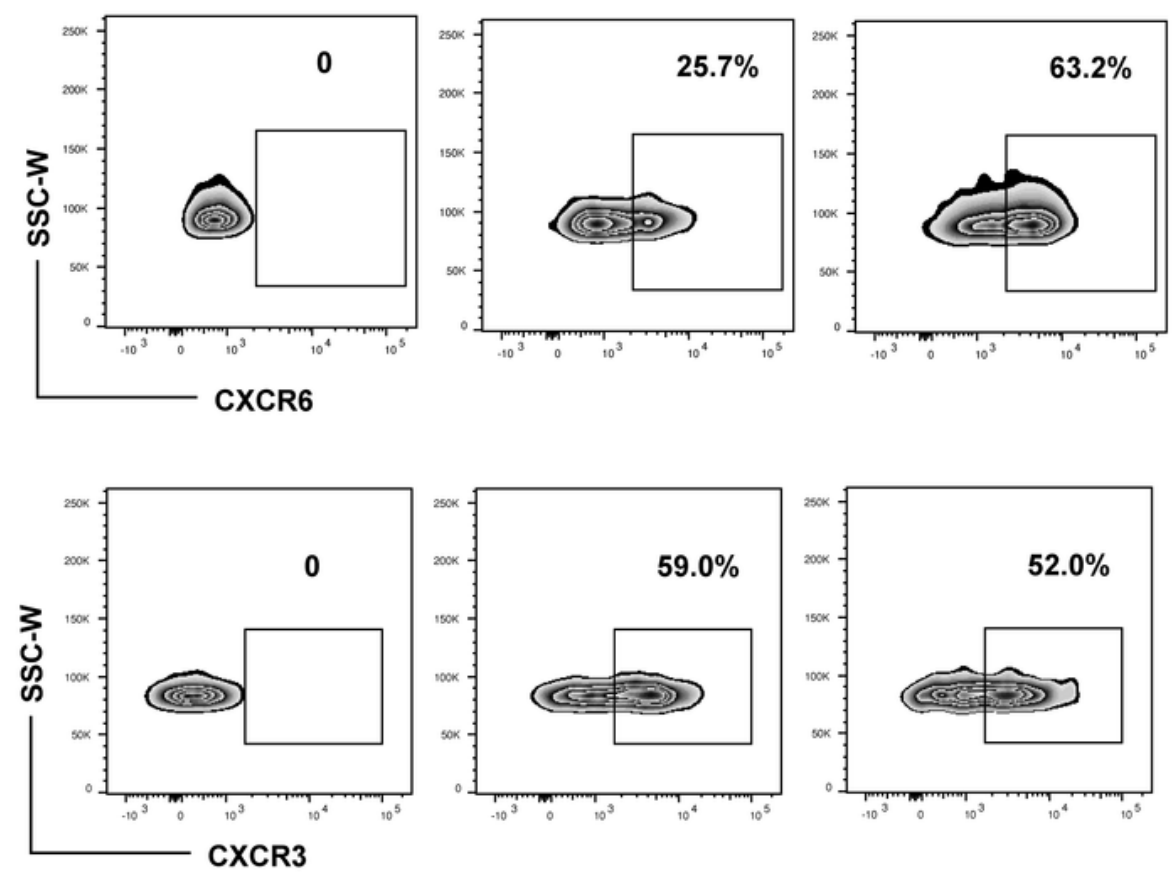

b

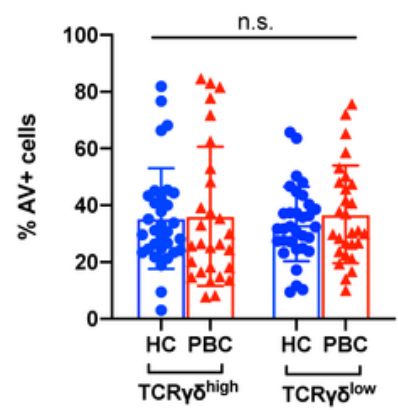

C

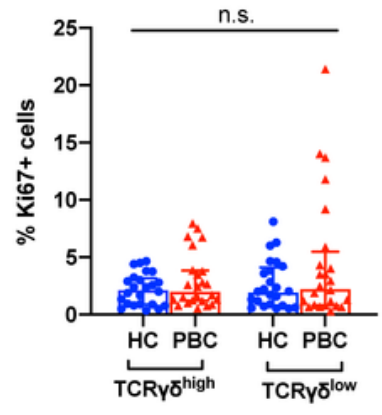

d

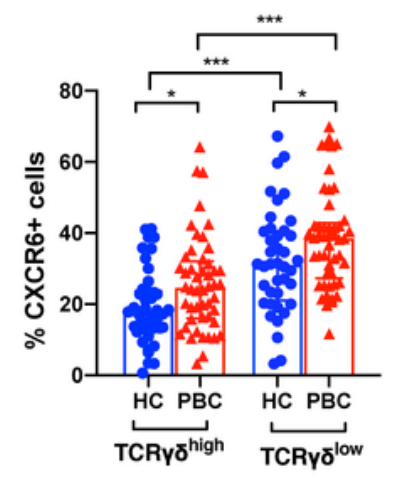

e

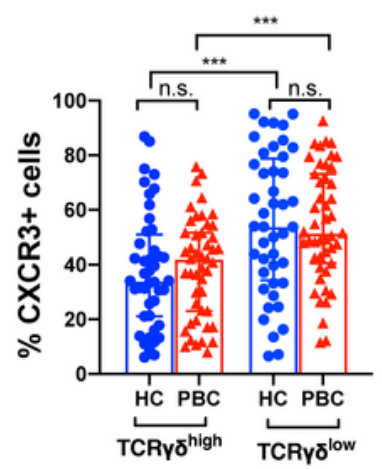

\section{Figure 3}

Alterations in apoptosis, proliferation, and the expression of liver-homing chemokine receptors in

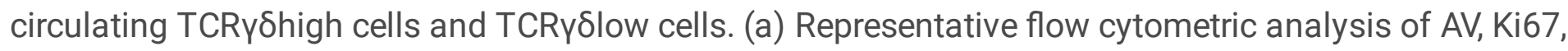
CXCR6 and CXCR3 expression in circulating TCRyठlow cells in HCs and PBC patients. (b) No difference

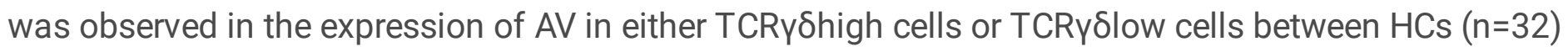

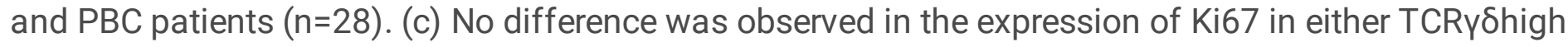


cells or TCRyठlow cells between HCs ( $n=24)$ and PBC patients $(n=24)$. (d) Increased expression of CXCR6

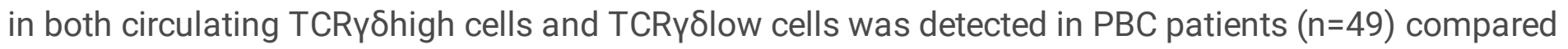
with HCs $(n=42)$. (e) No difference was observed in the expression of CXCR3 in either TCRyסhigh cells or TCRyolow cells between HCs $(n=44)$ and PBC patients $(n=47)$. Data are presented as medians with interquartile ranges. ${ }^{\star} P<0.05$, ${ }^{\star \star} P<0.01,{ }^{\star \star *} P<0.001$, n.s. $P>0.05$. AV, Annexin $V ; H C$ s, healthy controls; $\mathrm{PBC}$, primary biliary cholangitis.

a

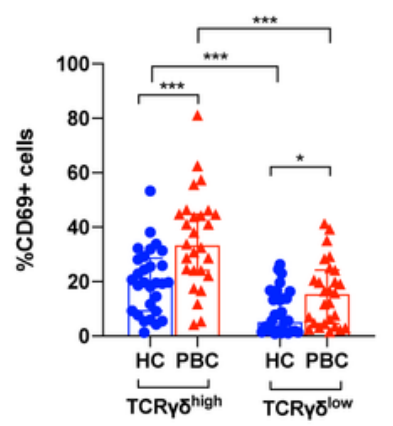

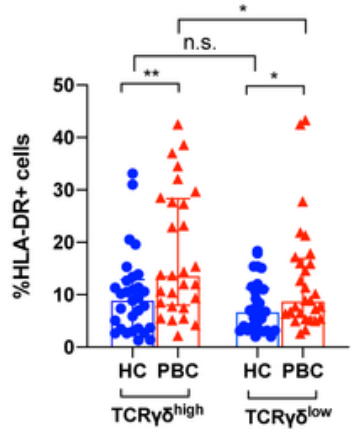

b

Gated on the CD3+TCRy $\delta^{\text {low }}$ subset

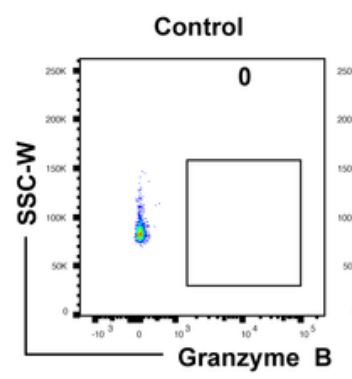

HC

PBC
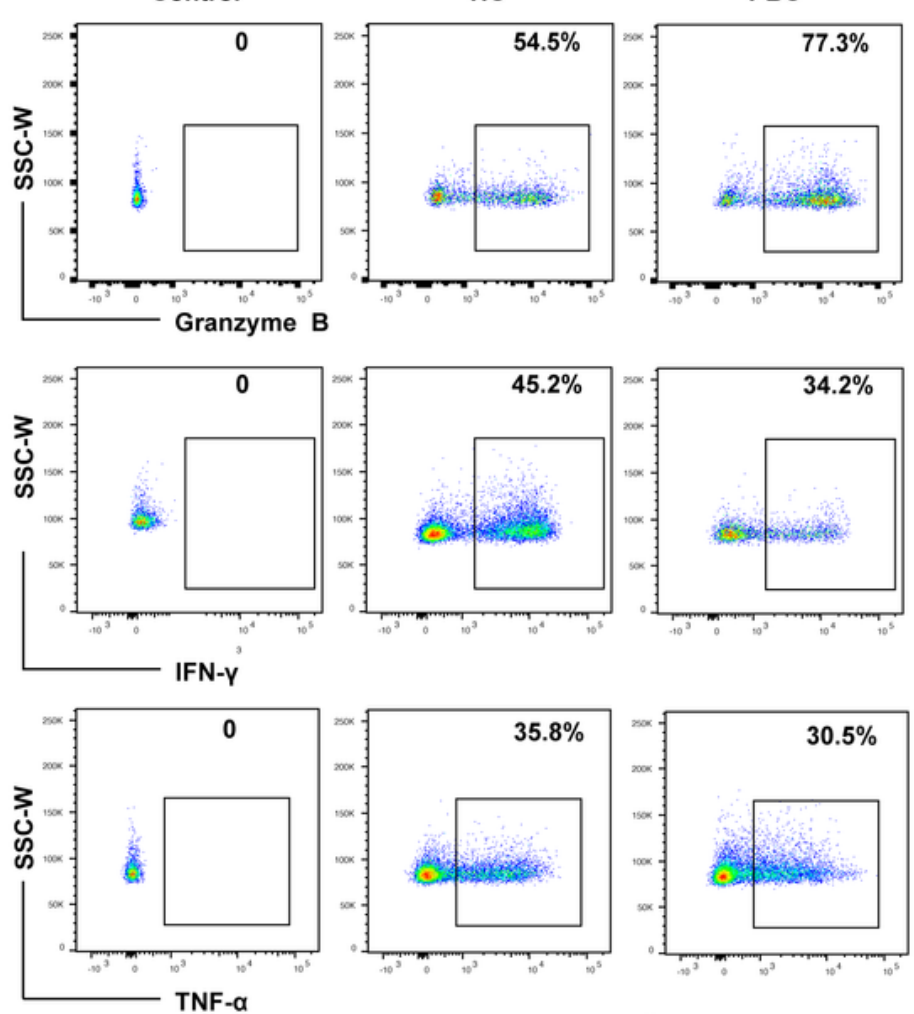

c
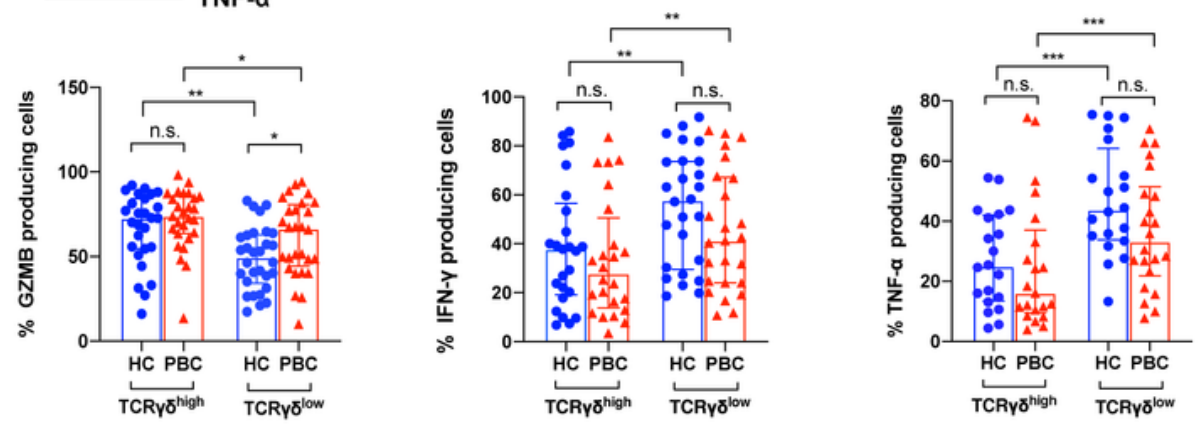

Figure 4 
Enhanced activation markers and altered cytokine production of circulating TCRyठhigh cells and

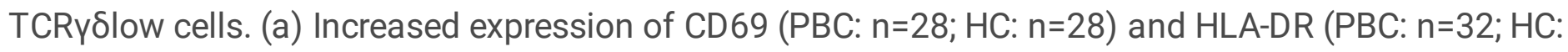

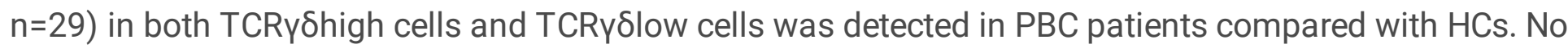

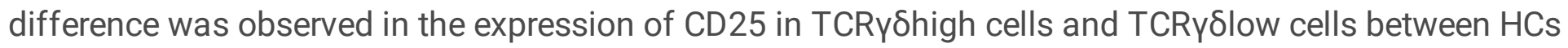
$(n=28)$ and PBC patients $(n=25)$. (b) Representative dot plot of cytokine production of TCRyסlow cells stimulated by PMA-ionomycin. (c) Increased expression of GZMB in circulating TCRyठlow cells was detected in PBC patients $(n=28)$ compared with HCs $(n=30)(P<0.05)$. No difference was observed in the expression of IFN-y (PBC: $n=25 ; H C: n=26)$ or TNF-a (PBC: $n=22 ; H C: n=20)$ in either TCRyठhigh cells or

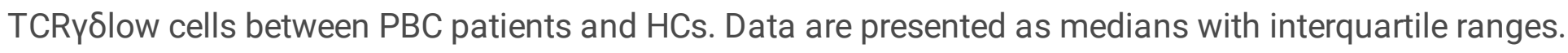
${ }^{\star} P<0.05$, ${ }^{\star \star} P<0.01$, ${ }^{* \star *} \mathrm{P}<0.001$, n.s. $P>0.05$. GZMB, granzyme $B ; H C s$, healthy controls; $P B C$, primary biliary cholangitis. 